\title{
Nonconvulsive Seizures and Dementia: A Case Report
}

\author{
Campana Chiara, Assenza Giovanni, Pellegrino Giovanni, Benvenga Antonella, \\ Assenza Federica, Ursini Francesca, Vernieri Fabrizio, and Tombini Mario
}

Dipartimento di Neurologia, Università Campus Biomedico, Via Alvaro del Portillo 200, 00128 Roma, Italy

Correspondence should be addressed to Assenza Giovanni, g.assenza@unicampus.it

Received 30 December 2010; Accepted 21 February 2011

Academic Editor: Giuseppe Curcio

Copyright (C 2011 Campana Chiara et al. This is an open access article distributed under the Creative Commons Attribution License, which permits unrestricted use, distribution, and reproduction in any medium, provided the original work is properly cited.

\begin{abstract}
Nonconvulsive status epilepticus (NCSE) is a severe medical condition that shows increased incidence in the elderly and is frequently underdiagnosed because of its pleomorphic presentation. We report an NCSE in a 76-year-old woman affected by dementia with acute change of cognitive status and behavior. Intravenous diazepam solved clinical and electroencephalographic manifestations. Neuropsychological assessment after NCSE conclusion showed impairment of several fields that remained unchanged at 3-month followup. NCSE should be considered when sudden and transient cognitive fluctuations appear in the elderly. Epileptic events in dementia occur frequently and are often underrecognized; this could be a misleading factor when considering a quick progression of mnesic performances. Moreover, recent findings both in animal models and in humans demonstrated the deep link between epilepsy and dementia, also supporting the hypothesis that epileptiform activity could contribute to cognitive impairment.
\end{abstract}

\section{Introduction}

Epileptic events, even a status epilepticus (SE), should be considered when fluctuations of cognition and awareness occur in elderly people, especially if dementia coexists $[1,2]$. In particular, the nonconvulsive status epilepticus (NCSE) is a pleomorphic condition, usually underdiagnosed, defined by the association of changes in consciousness without major motor signs and continuous or repeated epileptic dis-charges beyond 30 minutes on EEG [3]. It appears most often as clinical confusion, corresponding either to absence status (AS) or complex partial status epilepticus (CPSE) [4-9]. According to experimental and clinical observations that focal epileptic activity tends to generalize less often in old age, elderly people show an increased incidence of NCSE-in particular acute symptomatic CPSE-especially in association with neurodegenerative, cerebrovascular, or neoplastic disorders $[1,4-6,10]$.

Furthermore, several clinical and experimental data support a close linkage between pathophysiological processes sustaining epilepsy and cognitive impairment of dementia. In fact, both the ictal and postictal effects of the seizures themselves and the effects of the interictal epileptiform EEG dis- charges may have an impact on cognition [11]; on the other hand, patients with Alzheimer disease $(\mathrm{AD})$ and other types of dementias are at 5-10 fold increased risk of epilepsy compared to age-matched controls [12]. Finally, $\beta$-amyloid was recently demonstrated to favour epileptiform activity and cognitive deficits in transgenic mouse models of $\mathrm{AD}[13,14]$.

We report an NCSE in a 76-year-old woman affected by dementia.

\section{Case Report}

A 76-year-old woman was admitted to our department complaining of an acute worsening of cognitive status and a fluctuating level of consciousness. One year ago, she received a diagnosis of dementia. She has also been suffering from recurrent complex partial seizures for 15 years, and she was taking two antiepileptic drugs (valproicacid, $1000 \mathrm{mg} /$ die and oxcarbazepine, $600 \mathrm{mg} / \mathrm{die}$ ); the monthly seizure frequency was two. Moreover, she was suffering from hypertension and depression and received several medications (ticlopidine, sartan/hydrochlorothiazide $50+$ $12.5 \mathrm{mg}$, paroxetine $20 \mathrm{mg} / \mathrm{die}$ ). Finally, in her history, there was a nonspecified cerebrovascular accident 16 years ago. 
Her relatives defined her actual behaviour as different from the usual one, and they noted a fluctuating cognitive status in the last weeks. Upon examination, she looked distracted and vacant. She replied to questions only after a brief pause and with inadequate answers. She had trouble speaking, and she was uncooperative and disoriented. Some minutes later she presented a restriction of consciousness (fixed gaze and unresponsive to any kind of stimuli) with motor signs (oro-alimentary automatisms and jerking of right foot) lasting some minutes. Then, only persisted consciousness restriction.

The EEG examination that was performed during the episode showed a subcontinuous theta-delta activity with sporadic low-voltage spikes prevalent in the bilateral frontotemporal regions longer than 30 minutes from symptoms' onset, and thus leading to the diagnosis of CPSE (Figure 1). She was promptly given intravenous diazepam (5 mg) with resolution of clinical and electroencephalographic manifestations. After diazepam administration the patient fell asleep. The EEG recording revealed clear interictal epileptiform waves on the left frontotemporal area during stage 2 NREM sleep (Figure 2). Brain MRI performed on the fourth day showed a T2-weighted abnormal hyperintensity in the left insular cortex consistent with a vascular gliotic area. A severe impairment of memory and attention with minor deficits of language and praxia were evident at the neuropsychological assessment on the fifth day after CPSE. MMSE score was $12 / 30$. She was discharged with an increased dose of oxcarbazepine (1800 mg/die). Neuropsychological evaluation demonstrated an unchanged cognitive performance at 3-month followup.

\section{Discussion}

Transient events including syncope, episodes of inattention, or confusion are frequent in elderly people, mainly in patients affected by dementia $[1,2]$. In our patient, with a known cognitive impairment, the acute change in behavior and cognition, associated with a restriction of consciousness, leads to the suspicion of an epileptic event. The EEG provided evidence of an electroclinical pattern of CPSE that explained the patient's symptoms.

Rapid cognitive decline in demented patients could mimic several disorders: metabolic encephalopathy, prolonged postictal confusion, psychiatric disorders, substance intoxication, transient global amnesia, and transient ischemic attack [15]. NCSE should be included in the differential diagnosis, as a potentially treatable cause of altered awareness in the elderly. NCSE appears to have a gradual development of symptoms (impaired consciousness; "epileptic twilight state" with confusion, strange behavior, and automatisms). Therefore, its identification is usually difficult, especially when clinical manifestation only consists of mild changes in behavior. In our patient, the presence of preexisting epilepsy and dementia fortified the presumption of a status epilepticus (SE) $[1,6,8,16]$. Furthermore, she was in treatment with paroxetine that reduces the seizure threshold [17]. Several previous studies have demonstrated the frequency of a delayed diagnosis of an NCSE in elderly due to the absence of certain clinical parameters [18]. Husain and colleagues tried to define a profile of clinical features highly suggestive of NCSE (risk factors for seizures such as history of epilepsy, dementia and stroke, and impaired mental state) in order to select those patients who need an emergency EEG [19]. The EEG is fundamental to obtain an early diagnosis and should be promptly considered as a standard protocol in acute de novo confusion in these patients with high level of suspicion for NCSE [3]. Moreover, EEG findings are necessary to localize the epileptic focus and to distinguish a CPSE from an absence status, since in the first one, a rapid cessation is required due to prolonged episodes that may be accompanied by neurological deficits. Benzodiazepines are the most commonly recommended agents $[20,21]$. The aims of therapy when treating NCSE are the improvement of individual's cognitive function and to clear EEG tracing from epileptiform discharges $[6,15]$. Our patient's critical EEG definitely improved during treatment. No causative medical disorders were recognized at blood test. We supposed that the patient was affected by symptomatic focal epilepsy, and her poor antiepileptic therapy compliance in association to a lower seizure threshold was the cause of SE.

The hypothesis of an "epileptic pseudodementia" has been later confuted by cognitive assessment followup that proved a stationary neuropsychological impairment. The patient's relatives noted a very different behavior prior to admission. We could hypothesize that she might have experienced some other prolonged epileptic events that were not recognized. Animal models and some observation in humans have shown that NCSE could be responsible for long-term deficits also in neuropsychological field. How much morbidity adds ictal confusion to a preexisting cognitive impairment remains highly debated. The authors confirm the influence of causative medical disorders, the treatment, and perhaps the persistence of seizure activity in complications and sequels of NCSE $[6,15]$. Both ictal and interictal epileptiform EEG discharges could have an additional and independent effect on cognition. Nevertheless, some authors downsize their role except for cryptogenic partial epilepsies that seem to be associated with a higher risk of cognitive impairment [22]. In our case, the left frontotemporal epileptic discharges should be compatible with clinical features of language and memory impairment and well match with the insular vascular gliotic area revealed by MRI. Thus, it could be argued that previous stroke caused the ischemic insular lesion and then symptomatic epilepsy.

Finally, interesting recent studies have speculated on several mechanisms that could link epilepsy to cognitive decline and vice versa. Cordonnier and De Reuck [23, 24] reported that epileptic seizures, both early and lateonset, after stroke were independent predictors of newonset dementia. They speculated that preexisting vascular pathologies that may predispose to both epileptic seizures and new-onset dementia could be white matter changes, silent infarcts, or microbleeds. Alternatively, this could be due to an underlying preclinical degenerative disorder such as Alzheimer's disease. The recent findings in $\mathrm{AD}$ animal models of causal relationship between $\mathrm{A} \beta$-induced aberrant excitatory neuronal activity and cognitive decline raised 


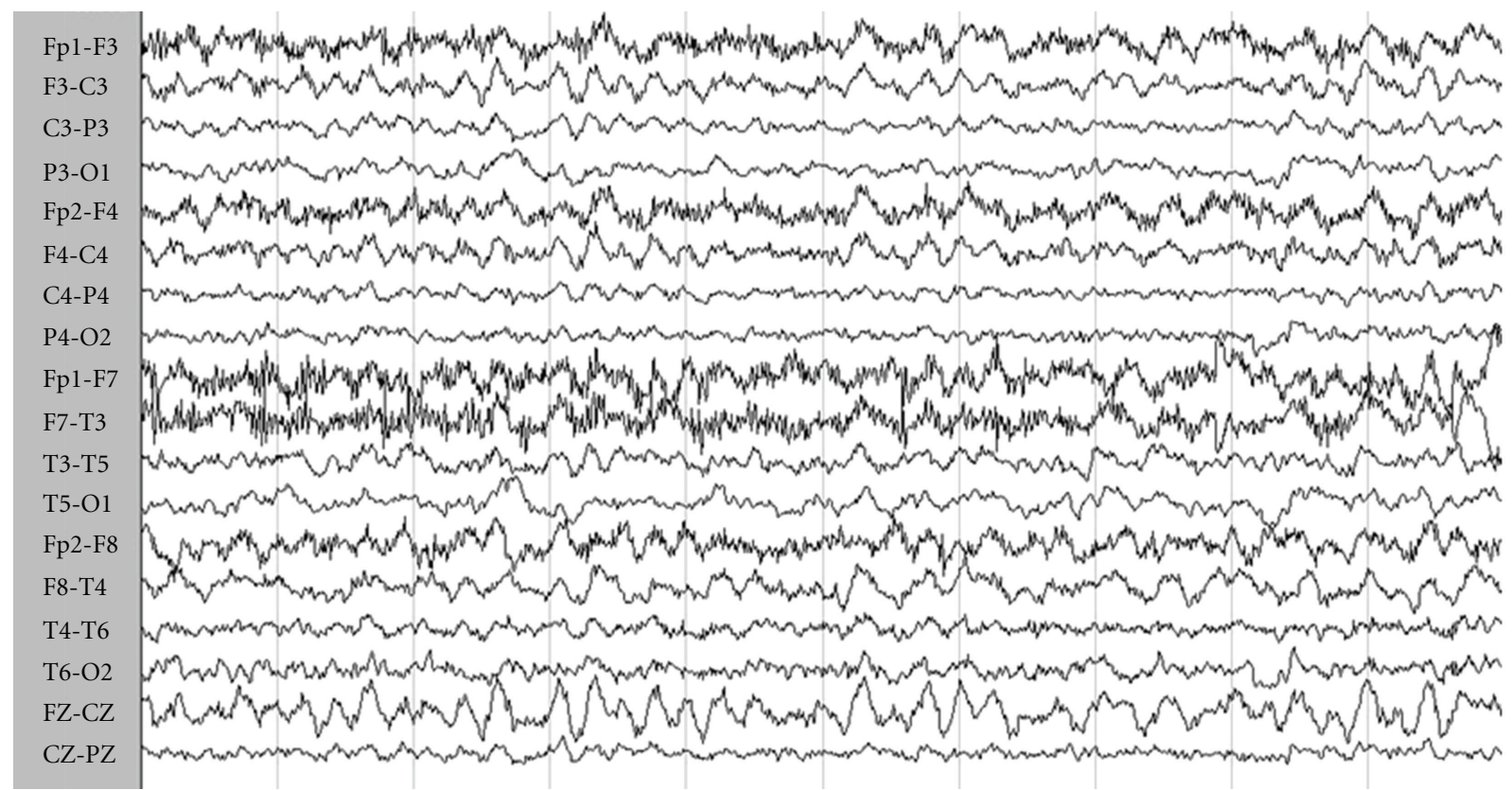

FIGURE 1: Ictal EEG. The EEG during the episode showed a subcontinuous theta-delta activity with sporadic low-voltage spikes prevalent in the bilateral frontotemporal regions.

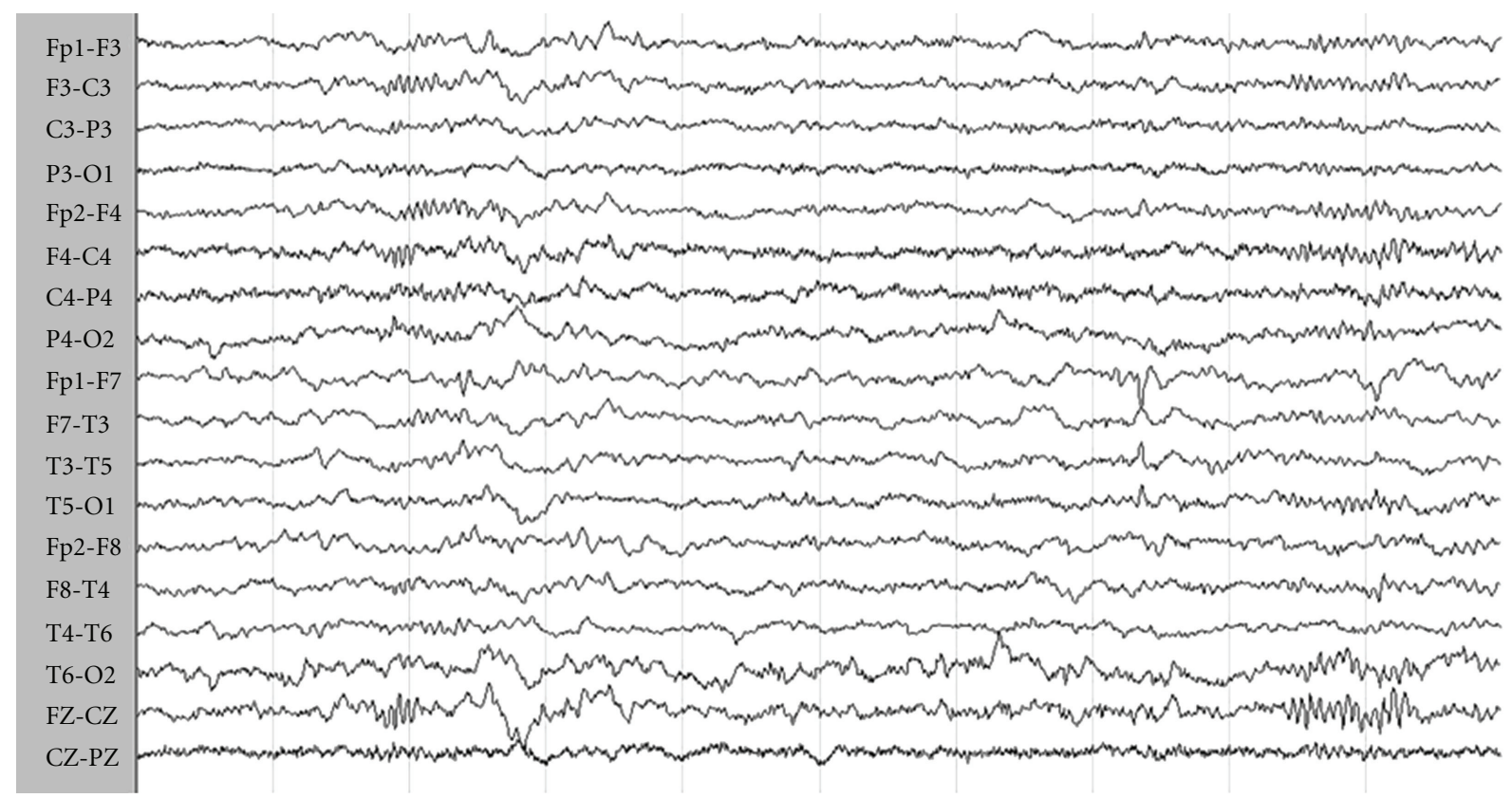

FIGURE 2: EEG activity after diazepam administration. After diazepam administration, the patient fell asleep, and EEG recording showed sharp waves on the left frontotemporal area during stage 2 NREM sleep.

the possibility that also in humans epileptiform activity could represent a primary mechanism that may contribute to cognitive deficits $[13,25,26]$. However, the long-term effect of seizure activity on the neurodegenerative disorder is unknown. Finally, the putative epileptogenic mechanism in patients with combined recurrent seizures and a progressive neurodegenerative disorder may relate to the findings of neuronal loss and gliosis involving selected regions such as the medial temporal lobe [25].

\section{Conclusion}

Epileptic events in dementia are frequent and often underrecognized, and this could be a misleading factor when 
considering a quick progression of mnesic performances. NCSE should be always considered when sudden and transient changes in behavioral or cognitive baseline condition are presented in an elderly patient, especially if affected by dementia when there are no other evident causes. It is mandatory to recognize the suggestive features for NCSE in an early stage and to perform a prompt EEG to diagnose and treat this epileptic condition.

\section{References}

[1] L. J. Stephen and M. J. Brodie, "Epilepsy in elderly people," Lancet, vol. 355, no. 9213, pp. 1441-1446, 2000.

[2] N. Scarmeas, L. S. Honig, H. Choi et al., "Seizures in Alzheimer disease: who, when, and how common?" Archives of Neurology, vol. 66, no. 8, pp. 992-997, 2009.

[3] F. J. Bottaro, O. A. Martinez, M. M. F. Pardal, J. E. Bruetman, and R. C. Reisin, "Nonconvulsive status epilepticus in the elderly: a case-control study," Epilepsia, vol. 48, no. 5, pp. 966972, 2007.

[4] M. Holtkamp, K. Buchheim, H. Siegmund, and H. Meierkord, "Optical imaging reveals reduced seizure spread and propagation velocities in aged rat brain in vitro," Neurobiology of Aging, vol. 24, no. 2, pp. 345-353, 2003.

[5] J. C. DeToledo, "Changing presentation of seizures with aging: clinical and etiological factors," Gerontology, vol. 45, no. 6, pp. 329-335, 1999.

[6] M. Walker, H. Cross, S. Smith et al., "Nonconvulsive status epilepticus: epilepsy research foundation workshop reports," Epileptic Disorders, vol. 7, no. 3, pp. 253-296, 2005.

[7] P.W. Kaplan, "Behavioral manifestations of nonconvulsive status epilepticus," Epilepsy and Behavior, vol. 3, no. 2, pp. 122-139, 2002.

[8] S. Haffey, A. McKernan, and K. Pang, "Non-convulsive status epilepticus: a profile of patients diagnosed within a tertiary referral centre," Journal of Neurology, Neurosurgery and Psychiatry, vol. 75, no. 7, pp. 1043-1044, 2004.

[9] W. Lennox, "The petit mal epilepsies: their treatment with tridione," Journal of the American Medical Association, vol. 129, pp. 1069-1073, 1945.

[10] J. L. Fernández-Torre and A. G. Díaz-Castroverde, "Nonconvulsive status epilepticus in elderly individuals: report of four representative cases," Age and Ageing, vol. 33, no. 1, pp. 78-81, 2004.

[11] A. P. Aldenkamp, J. Overweg, T. H. Gutter, A. M. Beun, L. Diepman, and O. G. Mulder, "Effect of epilepsy, seizures and epileptiform EEG discharges on cognitive function," Acta Neurologica Scandinavica, vol. 93, no. 4, pp. 253-259, 1996.

[12] M. F. Mendez and G. T. H. Lim, "Seizures in elderly patients with dementia: epidemiology and management," Drugs and Aging, vol. 20, no. 11, pp. 791-803, 2003.

[13] J. J. Palop, J. Chin, E. D. Roberson et al., "Aberrant excitatory neuronal activity and compensatory remodeling of inhibitory hippocampal circuits in mouse models of Alzheimer's disease," Neuron, vol. 55, no. 5, pp. 697-711, 2007.

[14] J. J. Palop and L. Mucke, "Epilepsy and cognitive impairments in Alzheimer disease," Archives of Neurology, vol. 66, no. 4, pp. 435-440, 2009.

[15] H. Meierkord and M. Holtkamp, "Non-convulsive status epilepticus in adults: clinical forms and treatment," Lancet Neurology, vol. 6, no. 4, pp. 329-339, 2007.
[16] P. W. Kaplan, "The clinical features, diagnosis, and prognosis of nonconvulsive status epilepticus," Neurologist, vol. 11, no. 6, pp. 348-361, 2005.

[17] F. Pisani, G. Oteri, C. Costa, G. Di Raimondo, and R. Di Perri, "Effects of psychotropic drugs on seizure threshold," Drug Safety, vol. 25, no. 2, pp. 91-110, 2002.

[18] R. D. Sheth, J. F. Drazkowski, J. I. Sirven, B. E. Gidal, and B. P. Hermann, "Protracted ictal confusion in elderly patients," Archives of Neurology, vol. 63, no. 4, pp. 529-532, 2006.

[19] A. M. Husain, G. J. Horn, and M. P. Jacobson, "Nonconvulsive status epilepticus: usefulness of clinical features in selecting patients for urgent EEG," Journal of Neurology Neurosurgery and Psychiatry, vol. 74, no. 2, pp. 189-191, 2003.

[20] H. Gastaut, P. Tinuper, U. Aguglia, and E. Lugaresi, "Treatment of certain forms of status epilepticus by means of a single oral dose of clobazam," Revue d'Électroencéphalographie et de Neurophysiologie Clinique, vol. 14, no. 3, pp. 203-206, 1984.

[21] F. W. Drislane, "Presentation, evaluation, and treatment of nonconvulsive status epilepticus," Epilepsy and Behavior, vol. 1, no. 5, pp. 301-314, 2000.

[22] A. P. Aldenkamp and J. Arends, "Effects of epileptiform EEG discharges on cognitive function: is the concept of "transient cognitive impairment” still valid?" Epilepsy and Behavior, vol. 5, supplement 1, pp. S25-S34, 2004.

[23] C. Cordonnier, H. Hénon, P. Derambure, F. Pasquier, and D. Leys, "Early epileptic seizures after stroke are associated with increased risk of new-onset dementia," Journal of Neurology, Neurosurgery and Psychiatry, vol. 78, no. 5, pp. 514-516, 2007.

[24] J. De Reucka, M. De Clercka, and G. Van Maeleb, "Vascular cognitive impairment in patients with late-onset seizures after an ischemic stroke," Clinical Neurology and Neurosurgery, no. 7, pp. 632-637, 2006.

[25] S. C. Rao, G. G. Dove, G. D. Cascino, and R. C. Petersen, "Recurrent seizures in patients with dementia: frequency, seizure types, and treatment outcome," Epilepsy and Behavior, vol. 14, no. 1, pp. 118-120, 2009.

[26] A. J. Larner, "Epileptic seizures in AD patients," NeuroMolecular Medicine, vol. 12, no. 1, pp. 71-77, 2010. 


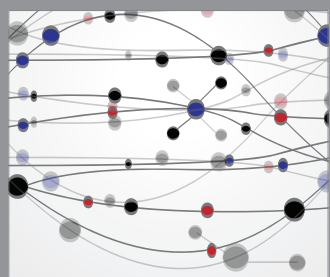

The Scientific World Journal
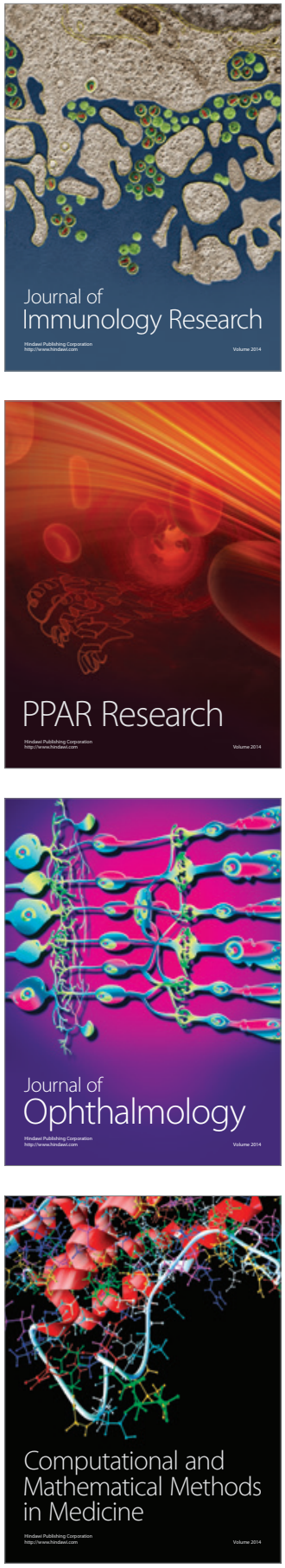

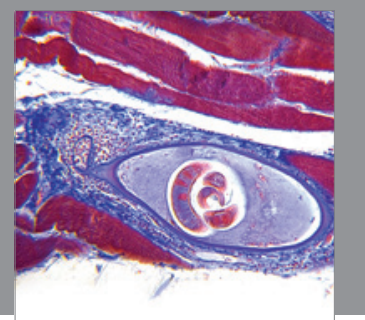

Gastroenterology

Research and Practice
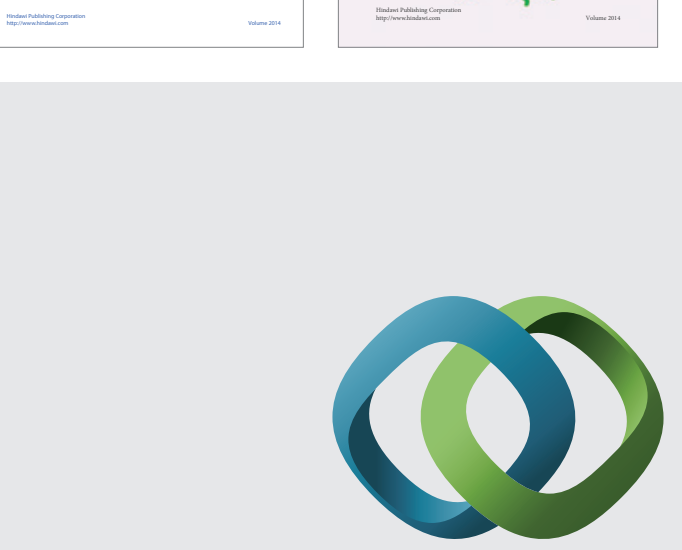

\section{Hindawi}

Submit your manuscripts at

http://www.hindawi.com
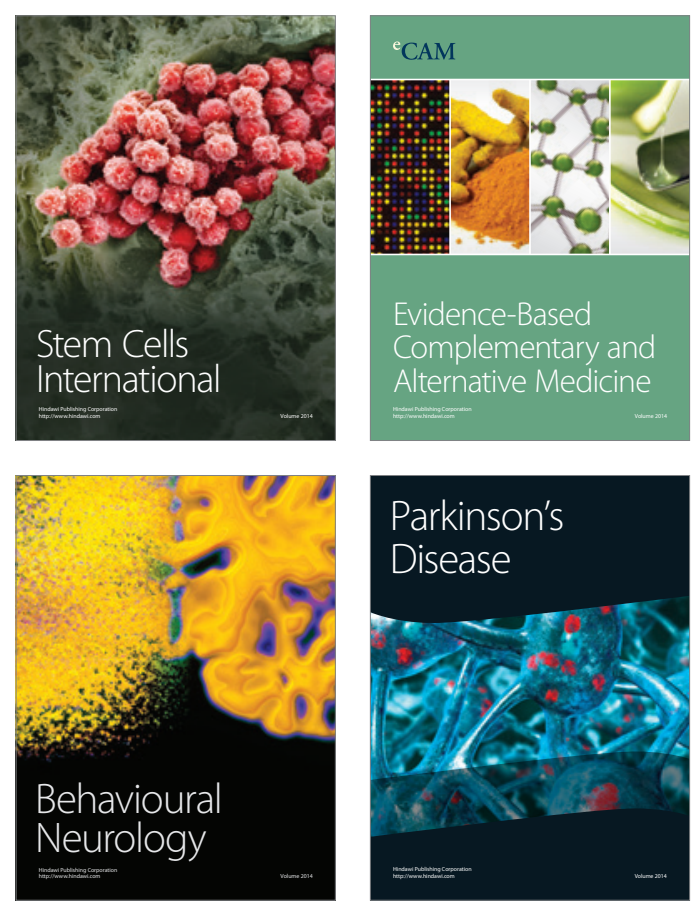

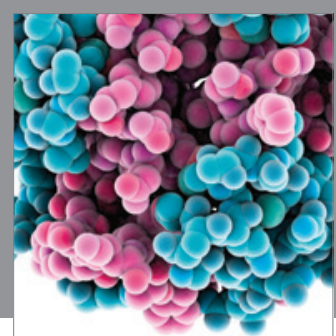

Journal of
Diabetes Research

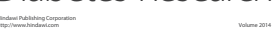

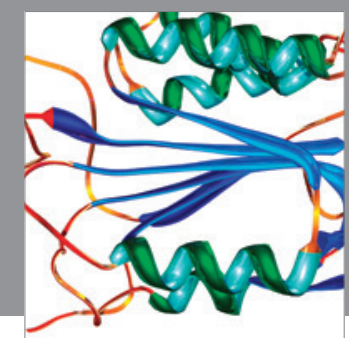

Disease Markers
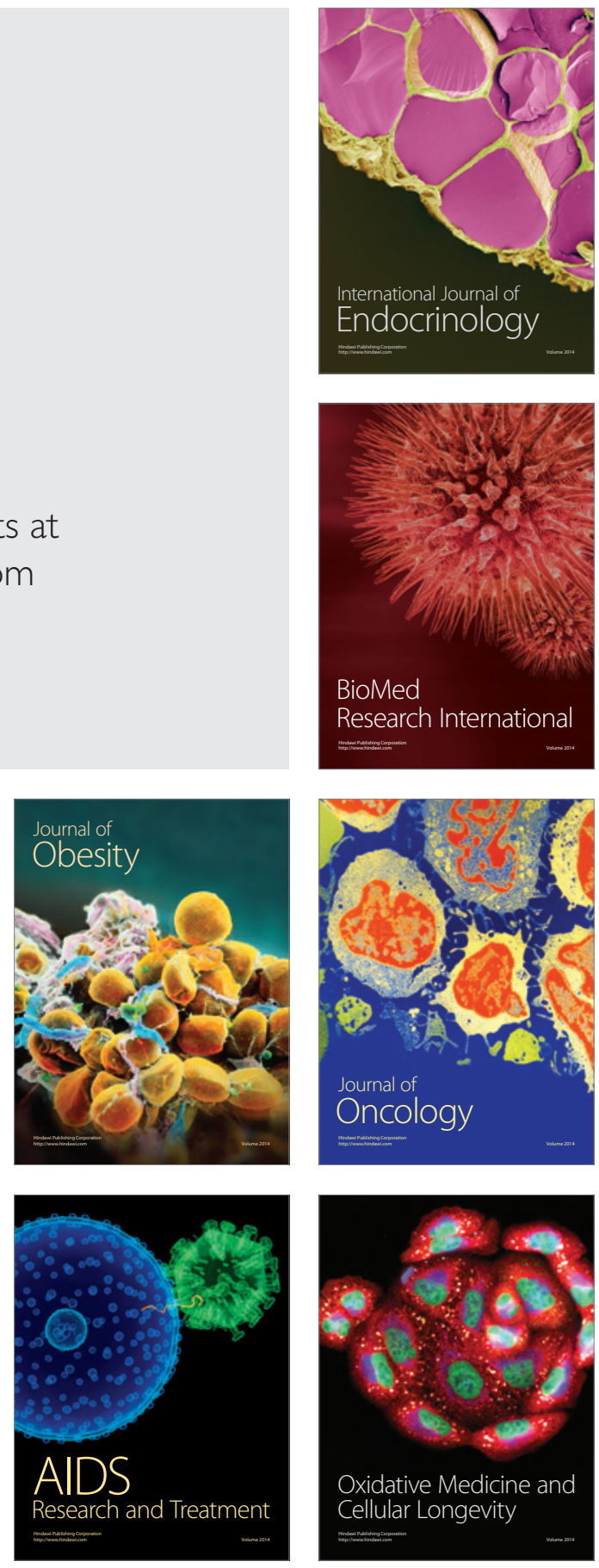